\title{
Encoding and Decoding Touch Location in the Leech CNS
}

\author{
Eric E. Thomson and William B. Kristan \\ Duke University, Durham, North Carolina 27710, and University of California, San Diego, La Jolla, California 92093
}

Spike times encode stimulus values in many sensory systems, but it is generally unknown whether such temporal variations are decoded (i.e., whether they influence downstream networks that control behavior). In the present study, we directly address this decoding problem by quantifying both sensory encoding and decoding in the leech. By mechanically stimulating the leech body wall while recording from mechanoreceptors, we show that pairs of leech sensory neurons with overlapping receptive fields encode touch location by their relative latencies, number of spikes, and instantaneous firing rates, with relative latency being the most accurate indicator of touch location. We then show that the relative latency and count are decoded by manipulating these variables in sensory neuron pairs while simultaneously monitoring the resulting behavior. Although both variables are important determinants of leech behavior, the decoding mechanisms are more sensitive to changes in relative spike count than changes in relative latency.

Key words: leech; local bend response; sensory coding; decoding; touch discrimination; latency

\section{Introduction}

The ability to respond differently to different stimuli, to discriminate among stimuli, is a behavioral aptitude whose importance is difficult to overstate. Without this skill, animals would maladaptively respond the same to potential mates, predators, and meals. The ability to discriminate where things are in space is a particularly important capacity: an animal typically orients toward or away from an object while interacting with it. Many psychophysical tasks probe how well organisms localize mechanical stimuli applied to the skin (Cholewiak, 1999). For instance, the "localization error threshold" measures the minimum discriminable distance between two tactile stimuli presented at different times (Cholewiak, 1999).

What is the neural basis of such discriminations? In particular, how do populations of sensory neurons encode stimulus location? Is the precise timing of spikes important, or does the nervous system encode location with the net number of spikes in a population of cells? In the past 50 years, many studies have investigated what features of spike trains in sensory afferents encode stimulus location. Single neurons in the somatosensory systems of rats (Petersen et al., 2001; Foffani et al., 2004), monkeys (Amassian, 1953), and humans (Johansson and Birznieks, 2004) encode touch location both by firing rates and response latency. Also, in humans, the relative onset time (i.e., the relative response latency) of responses in a population of primary sensory afferents accurately represents the shape of an object and direction of its force on the hand (Johansson and Birznieks, 2004).

Unfortunately, studies in which sensory responses are re-

Received Dec. 21, 2005; revised May 29, 2006; accepted June 21, 2006.

This work was supported by National Institutes of Health (NIH) Research Grants MH43396 and NS35336 to W.B.K. and by NIH Predoctoral Fellowship GM 08107 and a Merck Fellowship to E.E.T. We thank E. J. Chichilnisky and Kevin Briggman for comments on this paper.

Correspondence should be addressed to William B. Kristan, University of California, San Diego, 9500 Gilman Drive, Pacific Hall 324, La Jolla, CA 92093. E-mail: wkristan@ucsd.edu.

D01:10.1523/JNEUROSCI.5472-05.2006

Copyright $\odot 2006$ Society for Neuroscience $\quad 0270-6474 / 06 / 268009-08 \$ 15.00 / 0$ corded, but not manipulated, suffer from the decoding problem: they do not, by themselves, tell us whether the information encoded by the various features (such as precise timing of spikes) is actually used by the downstream networks to control behavior. Real, as opposed to ideal, decoders of the sensory response might not be sensitive to informative and precise spike timing, but could pool responses and guide behavior using the average activity in a population of sensory afferents (Parker and Newsome, 1998). We have used the medicinal leech (Hirudo medicinalis) (see Fig. $1 A$ ) to experimentally address the decoding problem because, (1) with no training, leeches respond to localized mechanical pressure by bending away from the location of stimulation, a behavior known as the "local bend response" (Kristan et al., 1982; Lewis and Kristan, 1998b; Baca et al., 2005), (2) a focal touch activates only two sensory neurons (see Fig. $1 B$ ) (Lewis and Kristan, 1998c) so we can record from all relevant sensory neurons while mechanically stimulating the skin, and (3) we can electrically stimulate the mechanoreceptors to generate arbitrary spike trains while we observe the resulting behavior.

\section{Materials and Methods \\ Physiology}

We dissected segments 9-11 from the leech as described previously (Baca et al., 2005) and excised the ganglia from segments 9 and 11, leaving only segment 10 innervated. For the encoding experiments, we pinned the section of body wall tightly onto a Sylgard-coated Petri dish, pulled ganglion 10 out from beneath the body wall (see Fig. $1 D$ ), and stabilized the ganglion with pins. To access the P-cell somata with our electrodes, we first removed the glial sheath covering the ventral surface of the ganglion. We recorded from the two ventral pressure $(\mathrm{P})$ cells in loose-patch configuration using a Getting amplifier, which operates in current-clamp mode. We acquired the data using pClamp 8 (Molecular Devices, Union City, CA) and all analyses were performed using Matlab (MathWorks, Natick, MA). We detected spikes off-line by setting a threshold above the noise. We used a servo-controlled force generator (Baca et al., 2005) to apply a $200 \mathrm{mN} / \mathrm{mm}^{2}$ pressure-step to the ventral surface of the body wall for $200 \mathrm{~ms}$ at one of five locations, stimulating a location five times before stimulating at another randomly selected location. For a given 
experiment, the five touch locations were either $-12^{\circ},-6^{\circ}, 0^{\circ}, 6^{\circ}$, and $12^{\circ}$, or $-6^{\circ},-3^{\circ}, 0^{\circ}, 3^{\circ}$, and $6^{\circ}$. Typically, each of the five locations was stimulated a total of 15 times, in pseudorandom order, in each preparation.

For the decoding experiments, we pinned out the body wall as described above, but loosely so that the body wall could move when the $\mathrm{P}$ cells were stimulated. The ventral $\mathrm{P}$ cells were monitored while in current-clamp mode using a loose patch recording configuration (Axoclamp 2B with a $10 \times$ head stage; Molecular Devices). At the beginning of the experiment, we determined the current thresholds required to evoke individual spikes in each P cell (thresholds ranged from 50 to $200 \mathrm{nA}$ ), and a $10 \mathrm{~ms}$ current impulse delivered at two to four times threshold reliably evoked single spikes in the P cells. We delivered stimuli every 3-7 min, depending on how long it took the body wall to relax after the previous trial. After stimulating the $\mathrm{P}$ cells, we captured images of the body wall between 40 and $125 \mathrm{~Hz}$ [SI-1280FM-CL camera (Silicon Imaging, Costa Mesa, CA) with XCAP image acquisition software (Epix, Buffalo Grove, IL)]. When we captured images at $>125$ frames per second, the movement between consecutive frames was too small to reliably estimate the movement (see below, Analysis, Decoding experiments).

\section{Analysis}

Encoding experiments. We quantified the neural response using four measures. Spike count is defined as the total number of spikes that occurred in the $200 \mathrm{~ms}$ period after body-wall stimulation. Latency is the difference in time between the first poststimulus spike and stimulus onset ( $\mathrm{P}$ cells do not fire unless the body wall is stimulated). The instantaneous firing rate (IFR) is defined as the reciprocal of the interspike interval. For instance, if two spikes occur twenty milliseconds apart, then the IFR between those two spikes is $1 / 0.02=50 \mathrm{~Hz}$. We applied principal components analysis (PCA; see below, Analysis, Decoding experiments, and Results) to the IFRs to reduce the dimensionality of the data and simplify our statistical analysis. We used as many principal components as were required to explain $99 \%$ of the variance in the data, and performed classification on the scores of the retained principal components (PCs). The initial IFR is the reciprocal of the time between the first two spikes in the neuronal response. We represented combinations of the different measures of the neural response (e.g., latency difference and count difference) as a two-element vector that contained the $z$-scores (Zar, 1999) of the variables in question.

The count difference, latency difference, and initial IFR difference are all defined as the value of the variable in the left $\mathrm{P}$ cell minus that in the right P cell; for example, the count difference on a given trial is the left $\mathrm{P}$-cell spike count minus the right $\mathrm{P}$-cell spike count.

To evaluate how well various neuronal variables discriminated touch location, we used an artificial neural network classifier, trained with a learning vector quantization algorithm (Kohonen, 1995) to calculate percent correct versus distance between touch locations (see Fig. $2 H$ ). The neural network, implemented in Matlab, contained two input units, two output units, and two hidden units. Through trial and error, we found that adding more hidden units did not improve performance. We used "leave one out" training, in which one trial was the test datum and the remaining trials were used to train the network. We applied the classifier to P-cell responses for all possible pairs of touch-location distances (e.g., in experiments in which we touched at $-6^{\circ},-3^{\circ}, 0^{\circ}, 3^{\circ}$, and $6^{\circ}$ there were four percent correct estimates for touch-location distances of $3^{\circ}$, three for distances of $6^{\circ}$, etc.).

To analyze behavioral discrimination of touch location (see Fig. $2 \mathrm{H}$, black line), we ran the same analysis on data used in a previous study (Baca et al., 2005). In those experiments, we touched the leech at five locations along the ventral midline on the body wall after the body wall was dissected out of the leech, as described above (see Physiology). Here, instead of estimating touch location based on P-cell response, we estimated touch location based on behavioral response (the quantification of behavior is described below in Analysis, Decoding experiments).

The exponential curves in Figures 2 and 4 were generated with a leastsquared error fit to the saturating exponential curve $0.5\left(1-\mathrm{e}^{\lambda x}\right)$ in Matlab, where $x$ is the touch location difference and $\lambda$ is a free parameter.
This function takes on a value of 0.5 (50\% correct) when $x$ is 0 and approaches $1.0(100 \%$ correct $)$ as $x$ is increased.

Decoding experiments. We derived the template of spike times to stimulate the $\mathrm{P}$ cells using data from the encoding experiments. For example, to deliver five spikes to a given $\mathrm{P}$ cell, we first calculated the mean IFR from all trials in the encoding experiments in which the $\mathrm{P}$ cell fired five spikes. The second spike was delivered at the mean interval between the first two spikes in all encoding trials when five spikes occurred in a P cell. We iterated this process until the times of all five spike times in the template were determined. We constructed templates of spike trains consisting of two to seven spikes from our encoding data.

Once we constructed the templates, we delivered $10 \mathrm{~ms}$ current pulses, as described above (see Physiology), at each spike time in the template. To generate latency differences, we delivered the templates to each P cell shifted in time by the appropriate amount (e.g., for a -10 ms latency difference, we began the left spike train template $10 \mathrm{~ms}$ before the right spike train template) (for example, see Fig. $3 A$ ).

The images acquired during P-cell stimulation were analyzed using optic flow estimation algorithms. We calculated the optic flow field between every pair of images in the image sequence captured while the leech responded to stimulation. Quantitatively, the optic flow field provides, for each pixel, a two-dimensional vector $x, y$ that represents how far the feature at that pixel moved between the two frames (see Fig. 3B). Hence, for $M$ captured images, there are $M-1$ flow fields. We represented the behavior of the leech between two frames with a bend profile, which is the mean movement of the body wall in the longitudinal direction (see Fig. $3 C$ ). The set of $M-1$ bend profiles after P-cell stimulation are represented as a surface plot, which shows the evolution of the behavior in time (see Fig. $3 D$ ). A detailed description of this analysis is given by Baca et al. (2005).

To more compactly describe the behavior over time, we performed PCA on the bend response matrices [specifically, we performed PCA on the set of poststimulus bend profiles (see Fig. 3D) after the profiles had been concatenated to form a single vector]. We included enough principle components to explain at least $90 \%$ of the variability in the behavioral data (note this is different from the $99 \%$ criterion used for the IFR). We extensively discuss the use of PCA to analyze local bending in Baca et al. (2005).

Classification of behavioral responses, discussed next, was performed on the scores of the retained principal components. The score of a PC for a given behavioral trial is the scaling factor of that $\mathrm{PC}$ required to reconstruct the original data. Responses that are far apart in PC score space are also far apart in the original high-dimensional data representation (i.e., they represent very different behaviors of the leech). Hence, representing the data in PC score space makes both statistical analysis and visualization of the data more tractable; typically only three components are required to capture most of the variability in our data (for example, see Fig. 5B).

We quantified behavioral sensitivity to changes in P-cell latency and count difference by building a classifier that estimated P-cell activity from the behavioral response, which was represented using PCA. Specifically, we used a linear discriminant analysis to find a "decision plane" that separates the data into two groups that correspond to the two possible spike train templates used to stimulate the P cells (Duda et al., 2000). For instance, one side of the plane might correspond to the behaviors generated by a spike-count difference of three spikes, the other side of the plane to those generated by a spike-count difference of minus two spikes. Technically, this decision plane lies perpendicular to the linear discriminant, which is the line along which between-group variability is maximized and within-group variability is minimized when the behavioral data are projected on that line (Duda et al., 2000). Just as with the encoding discrimination, we used "leave one out" training to build the discriminant, and the test data point was then classified using the learned discriminant. This process was applied to all $n$ data points, and the percentage of correctly classified trials gives the percent correct for the given comparison.

Because the number of trials in the decoding experiments was sometimes small (between 16 and 40 trials), we used a jackknife (Efron and Tibshirani, 1994) estimate of the mean percent correct for each experi- 
A.

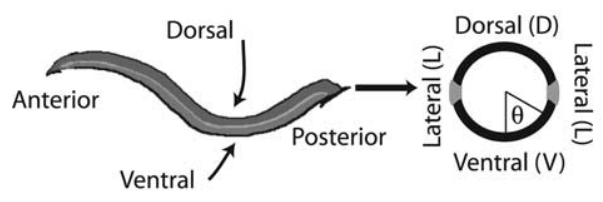

B.
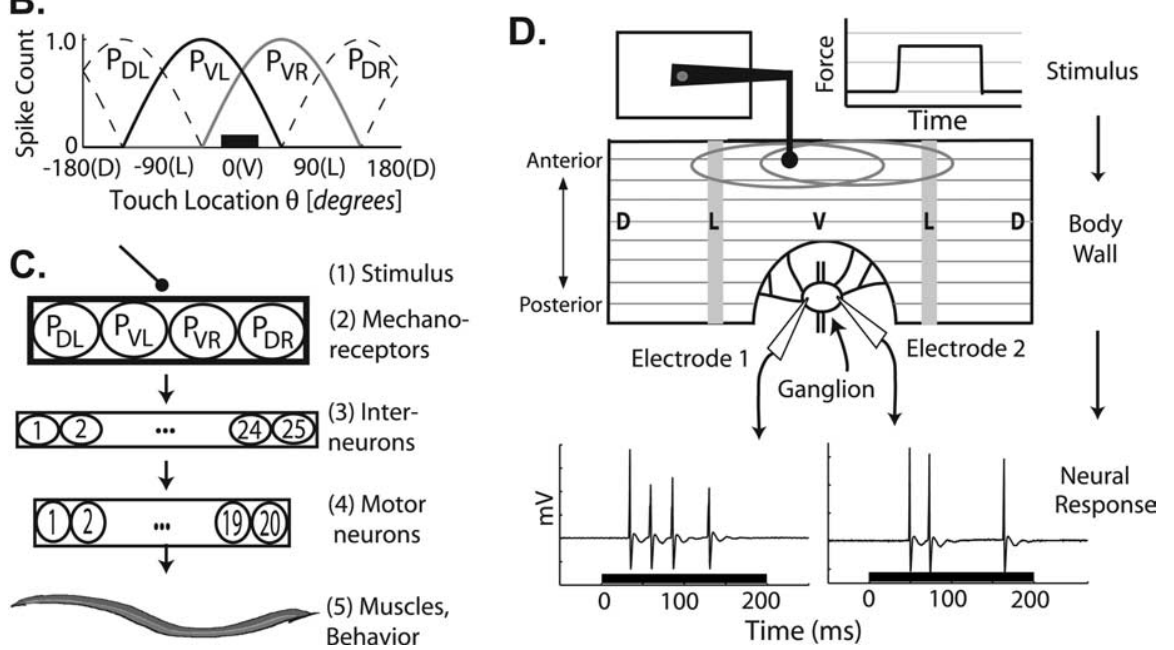

Figure 1. Background and methods for the encoding experiments. $A$, Left, lllustration of a leech. The leech is a segmented worm with dorsoventral (top to bottom) and anteroposterior axes (front to back, also known as the longitudinal axis). Right, Illustration of a cross section of a leech showing the circumferential axis of its body wall. The ventral midline of the leech (V) is taken to be the origin $\left(\theta=0^{\circ}\right)$. $\boldsymbol{B}$, Receptive fields show spike count in the four $\mathrm{P}$ cells versus touch location $\theta$ along the circumferential axis (adapted from Lewis and Kristan, 1998c). The spike counts are normalized to the maximum firing rate for each neuron (V ventral; D, dorsal; $L$, left; $R$, right). The black bar shows the region where we stimulated the leech. $C$, Schematic of the local bend network (see Results for description). $\boldsymbol{D}$, In the encoding experiments, we delivered a force step (top) to the leech body wall (middle) while recording voltages from the two ventral $P$ cells (bottom). The extent of the receptive fields of the cells is indicated by ovals on the body wall, and the horizontal lines represent annulus borders (each segment of the leech is made up of five raised ridges known as annuli). The black bars on the time axis of the voltage traces indicate the time course of the force step.

ment. We did not bias-correct the jackknife estimates because, in numerical simulations, we found that such corrections actually biased our percent correct estimates upward.

\section{Results}

\section{Encoding Experiments}

A relatively simple three-layered network of $\sim 50$ neurons mediates the local bend response (Fig. 1C). The response is initiated by activity in four pressure-sensitive mechanoreceptors known as $\mathrm{P}$ cells (Fig. 1 B) (Kristan, 1982; Lewis and Kristan, 1998c; Zoccolan and Torre, 2002), which excite $\sim 25$ interneurons (Lockery and Kristan, 1990; Lewis and Kristan, 1998a), which in turn excite $\sim 20$ motor neurons that synapse onto each other as well as the muscles in the leech body wall (Kristan, 1982; Lockery and Kristan, 1990).

Previous studies have shown that $\mathrm{P}$-cell spike counts vary with touch location along the circumference of the leech body wall (Fig. 1 B) (Kristan, 1982; Lewis and Kristan, 1998c). To determine whether latency also encodes touch location, we recorded responses in the two ventral P cells while delivering pressure steps to the ventral surface of the leech body wall (Fig. 1D). We delivered the stimuli close to the ventral midline $(\theta=0)$ to activate only the two ventral P cells (Fig. 1 B). We applied pressure for 200 $\mathrm{ms}$ because previous work has shown that the maximal encoding accuracy using P-cell spike counts is reached within $200 \mathrm{~ms}$ of stimulus onset (Lewis and Kristan, 1998c). In all 1200 trials from eight leeches in the current experiments, stimulation evoked spikes in both ventral P cells (Fig. 2A).
The P-cell response latency significantly increased with distance from the receptive field center in 16 of 16 cells, whereas spike count significantly decreased in nine of the $16 \mathrm{P}$ cells $(\alpha=0.05$; ANOVA) (Zar, 1999). The initial firing rate, calculated as the reciprocal of the first interspike interval (see Materials and Methods), significantly decreased with distance from the receptive field center in 10 of $16 \mathrm{P}$ cells. The first interspike interval, the shortest time period to estimate the firing rate, can potentially encode touch location more accurately than the spike count because it is a continuous variable (Johansson and Birznieks, 2004). These trends are illustrated for a single $P$ cell in Figure $2 B-D$.

The relative latency between the two $\mathrm{P}$ cells was also strongly correlated with touch location; when the stimulus was to the left of the ventral midline, the first spike in the left $\mathrm{P}$ cell occurred before the first spike in the right $\mathrm{P}$ cell. Conversely, the right $\mathrm{P}$ cell responded first to touches to the right of the ventral midline. The two $\mathrm{P}$ cells began to fire action potentials nearly simultaneously when the leech was touched at the ventral midline (Fig. 2A). Quantitatively, the latency difference, defined as the response latency in the left $\mathrm{P}$ cell minus the response latency in the right $P$ cell, was significantly correlated with touch location (Fig. 2E) in all eight leeches tested (maximum $p$ value, $10^{-5}$; ANOVA). On average, for a touch location change of $1^{\circ}$ on the body wall, the latency difference shifted by $1.2 \mathrm{~ms}$ (SEM, 0.2).

The spike count and initial firing-rate differences were also correlated with touch location, with more spikes (and a higher initial firing rate) in the $\mathrm{P}$ cell that was stimulated closer to its receptive field center (Figs. $2 F, G$ ). This correlation was significant in some leeches (two of four for counts; one of four for rates) when touch locations were $3^{\circ}$ apart, and in all leeches when the touch locations were $6^{\circ}$ apart ( $\alpha=0.05$; ANOVA). On average, for a touch location change of $1^{\circ}$ on the body wall, there was a change of -0.13 in the spike count difference between the $P$ cells (SEM, 0.02) and a change of $-1.2 \mathrm{~Hz}$ in the initial firing rate difference (SEM, 0.24).

In all eight leeches, the correlation coefficient between touch location and latency difference was higher than the correlation coefficient between touch location and both initial firing rate and count differences. Surprisingly, the correlation coefficients measured for initial firing rate difference and count difference with touch location were not statistically different ( $p=0.59 ; t$ test).

We then quantified how well the latency difference discriminated touch location. For all responses to stimulation at two locations in a given preparation (e.g., the latency differences in response to stimulation at $-6^{\circ}$ and $0^{\circ}$ ), we used a neural network classifier to estimate which stimulus location generated a given response (see Materials and Methods). Figure $2 \mathrm{H}$ illustrates the percentage of correctly classified trials as a function of touch location separation. Percent correct measures the separation between the two latency-difference distributions produced when 
the body wall is touched at two different locations (Thomson and Kristan, 2005). We fit percent correct versus touch location separation with a saturating exponential function, the neurometric function (Fig. 2H, red line) (Britten et al., 1992). We applied the same procedure using count difference (Fig. $2 \mathrm{H}$, blue line) and initial firing rate difference (Fig. $2 \mathrm{H}$, green line).

Latency difference proved to be the best discriminator of touch location: its neurometric function rose markedly faster than either of the other two. Quantitatively, the distribution of percent correct values using latency difference was significantly different from the distributions for count difference and for initial firing rate difference $[p<0.001$; Kolmogorov-Smirnoff test (Zar, 1999)]. The distributions for count difference and initial firing rate difference were statistically indistinguishable ( $p=0.97$; KolmogorovSmirnoff test). The behavioral discrimination of the leech, calculated using data from previous experiments (Baca et al., 2005) is included for comparison (Fig. $2 \mathrm{H}$, black line).

We verified that by including only the initial instantaneous firing rate, we were not missing information contained in the entire time-varying instantaneous firing rate of the P cells (see Materials and Methods). The neurometric function obtained using the difference between the full timevarying instantaneous firing rates of the $\mathrm{P}$ cells were not significantly different from that using only the initial firing rate difference or count difference ( $p>0.15$ in each case; Kolmogorov-Smirnoff test).

Because the neurometric function represents the discrimination performance over a wide range of stimuli, to summarize the overall performance we used a standard measure of discrimination threshold, namely the touch-location separation at which the classifier achieved 75\% correct (Johnson and Phillips, 1981). We used this as a measure of the touch location discrimination threshold (i.e., the minimum discriminable touch location difference). Latency differences discriminated touch locations that were $4^{\circ}$ apart, count differences discriminated touches $13^{\circ}$ apart, whereas the leech behaviorally discriminates touch locations $9^{\circ}$ apart (Fig. $2 H$ ).

(In a typical leech with a $1.5 \mathrm{~cm}$ circumference, $4^{\circ}$ corresponds to a distance of $<200 \mu \mathrm{m}$.) Hence, latency difference discriminates touch locations over three times better than count difference and two-fold better than the behaving leech.

Combining the different measures of the neural response did not improve discrimination. As described in Materials and Methods, we combined the different variables by forming vectors of
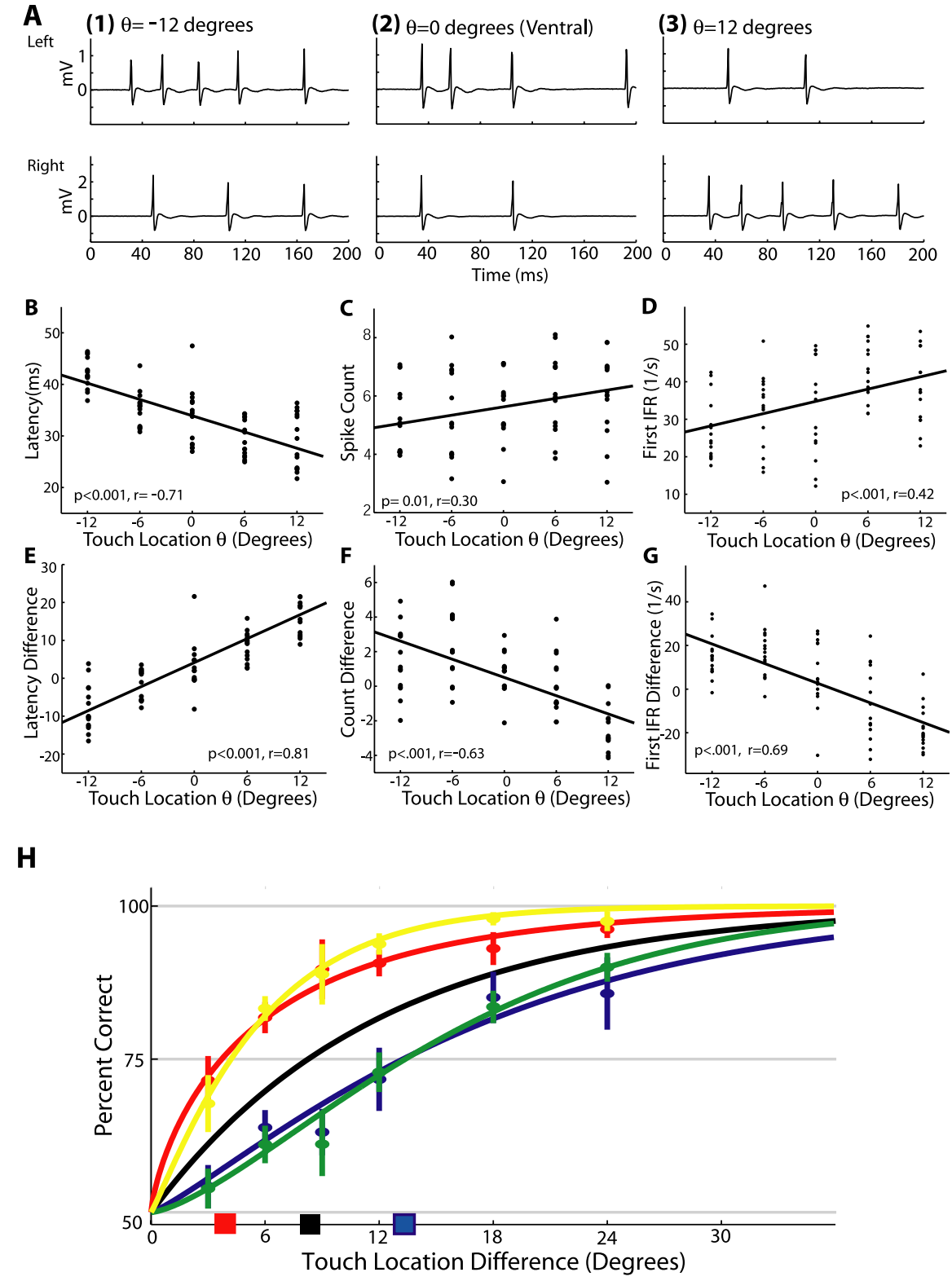

Figure 2. Results of encoding experiments. $\boldsymbol{A}$, Representative neural responses when the body wall is touched $12^{\circ}$ to the left of the ventral midline $(\boldsymbol{A} \mathbf{1})$, at the ventral midline $(\boldsymbol{A 2})$, and 12 degrees to the right of the ventral midline $(\boldsymbol{A} \mathbf{3})$. The top panels are responses in the left $P$ cell, and the bottom panels are from the right $P$ cell. $\boldsymbol{B}-\boldsymbol{D}$, Scatter plots of response latency, spike count, and initial firing rate versus touch location for the right $P$ cell from a single experiment ( $n=60$ trials). Lines are the best linear fits. In these and the following panels, $r$ is the correlation coefficient and $p$ is the $p$ value when the null hypothesis is that the variables are not correlated (ANOVA). $\boldsymbol{E}-\mathbf{G}$, Scatter plots of latency difference (latency in left P cell minus latency in right $P$ cell), count difference (count in left $P$ cell minus count in right $P$ cell), and initial firing rate difference (initial firing rate in left $P$ cell minus initial firing rate in right $P$ (ell) versus touch location. Data are from the same experiment as in the previous panels. $\boldsymbol{H}$, Percent correct as a function of touch location difference (filled circles with error bars representing mean \pm SEM, with the averages defined over all experiments). Red, Latency difference; blue, count difference; green, initial firing rate difference; yellow, latency difference and initial firing rate difference combined; black dashed line, behavior. The lines are the corresponding neurometric functions (see Results, Encoding experiments). The filled squares at the bottom indicate the touch location difference at which the classifier reached $75 \%$ correct $\left(4^{\circ}\right.$ for latency difference and latency difference combined with initial firing rate difference, $13^{\circ}$ for count difference as well as initial firing rate difference, and $9^{\circ}$ for behavior).

their $z$-scores so they would have the same means and SDs. Combining latency and count differences showed a slight improvement at larger separation between touch locations (Fig. $2 \mathrm{H}$, yellow line), but when compared over the full range of touch location differences, the performance was not significantly improved ( $p=0.91$; Kolmogorov-Smirnoff test). Similarly, combining latency with initial firing rate or the time-varying firing 
rate produced insignificant change in discrimination $(p>0.3$ in each case; Kolmogorov-Smirnoff test). This lack of improvement may result from the lack of independence among the measures. For instance, latency difference and count difference had a significant (negative) correlation in seven of eight leeches (three of four when the touch locations were $3^{\circ}$ apart and four of four when touched $6^{\circ}$ apart; ANOVA, $\alpha=0.05$ ).

Despite such correlations between variables, each variable continued to track touch location on those trials in which the other variable did not change. For example, in those 94 of 600 trials $(16 \%)$ in which the two P cells generated the same number of spikes (i.e., their count difference was 0 ), the latency difference varied by up to $30 \mathrm{~ms}$ and was still strongly correlated with touch location ( $p<0.001$; ANOVA). Similarly, when we analyzed those trials with nearly identical latency difference (i.e., the absolute value of the latency difference was $<2 \mathrm{~ms}$ ), the count difference varied by as much as three spikes and was significantly correlated with touch location ( $p=0.01$; ANOVA).

\section{Decoding experiments}

To determine how well the local bending circuit decodes latency difference and count difference, we stimulated the two ventral P cells with trains of brief current pulses while acquiring image sequences of the behaving body wall (Fig. $3 A$ ). We used patterns of $\mathrm{P}$-cell spikes that were based on firing rate statistics observed in the encoding experiments (see Materials and Methods). We calculated the optic flow field between every pair of images in each image sequence (Fig. 3B). The optic flow field describes the movement of the body wall between each consecutive pair of images in a sequence (see Materials and Methods). From the optic flow field, we extracted the bend profile (i.e., the mean longitudinal movement of the body wall as a function of location on the circumferential axis of the body wall) (Fig. 3C) (Baca et al., 2005). We characterized the full behavioral response after $\mathrm{P}$-cell stimulation as a sequence of such bend profiles over time (Fig. 3D).

\section{Varying spike-count differences}

We first varied count difference between the two ventral P cells while keeping their latency difference fixed. For example, in one experiment, changing the count difference by four spikes produced a clear difference in the behavioral response (Fig. $4 A$ ). In 27 such experiments, we changed the count difference between one and six spikes. (This is twice the range of three spikes seen in response to mechanical stimulation at a given latency difference.)

We then quantified how sensitive the leech was to such changes in spike-count difference, that is, how well the leech behaviorally discriminates such changes. We used the same procedure as that used to quantify how well the P cells discriminate touch location. That is, we used a classifier to estimate the stimulus applied (i.e., the count difference used) from the behavioral data for each trial. If the behavior is clearly dissimilar for the two count differences in a given experiment, then the classifier, based only on the behavioral data, will be able to correctly predict which count-difference was applied on each trial.

Local bending proved to be exquisitely sensitive to changes in count difference: a count difference change of just one spike was discriminated well above chance $(71 \%$ correct; $p<0.001$, onesided $t$ test when compared with $50 \% ; n=11)$. The discrimination function (Fig. $4 B$ ) shows that the threshold count difference change (i.e., $75 \%$ correct) was 1.8 spikes. From the encoding results, we know that a count difference change of 1.8 spikes corresponds to a touch location change of $14^{\circ}$ (this follows from

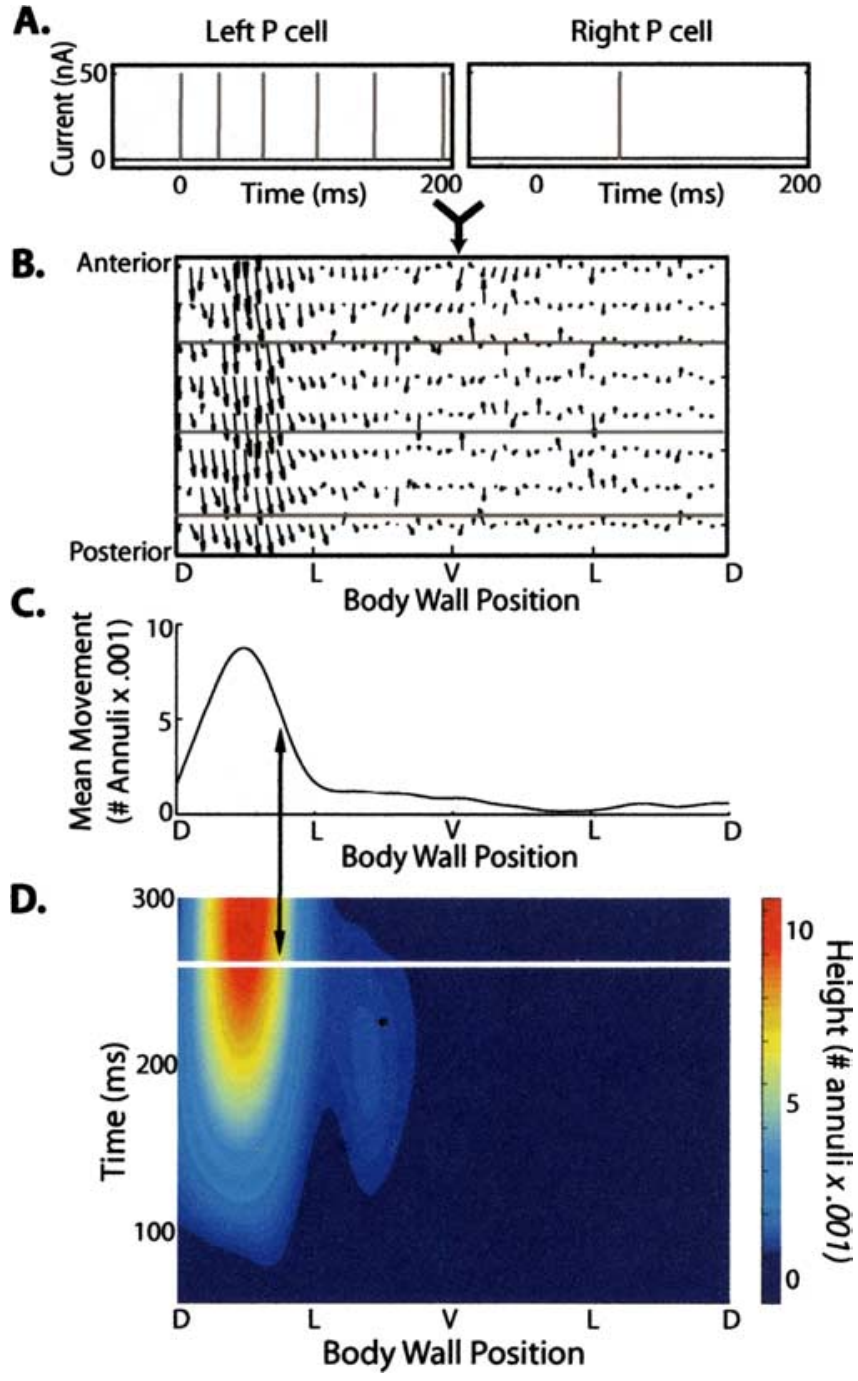

Figure 3. Methods used in the decoding experiments. $\boldsymbol{A}$, We delivered brief (10 ms) current pulses to the left and right ventral $P$ cells such that each pulse generated one action potential. In the example shown, six spikes were evoked in the left $P$ cell beginning at $t=0 \mathrm{~ms}$ and a single spike was evoked in the right $\mathrm{P}$ cell at $t=75 \mathrm{~ms}$. $\boldsymbol{B}$, An optic flow field, which represents the movement (at each pixel) between two consecutive images of the body wall. This example shows the flow field between images captured at 270 and $280 \mathrm{~ms}$ after onset of $P$ cell stimulation. Note the movement is clearly centered to the left of the ventral midline. Horizontal gray lines represent annulus borders. $C$, The bend profile, which is the mean body wall movement in the longitudinal direction (the $Y$ direction) calculated from the flow field in $\boldsymbol{B}$. D, A complete representation of the longitudinal component of the local bend response on a single trial: the set of bend profiles at 10 ms intervals from 0 to $300 \mathrm{~ms}$ after stimulation of the $P$ cells. The white horizontal line indicates the time slice shown in $\boldsymbol{C}$.

the observation in the encoding experiments that a change of touch location of $1^{\circ}$ causes a change of 0.13 in the count difference).

\section{Varying latency differences}

We next varied the latency difference between the two P cells while holding the count difference constant (Fig. 4C). We performed 53 such comparisons, varying the latency difference from 6 to $200 \mathrm{~ms}$. (In response to mechanical stimulation, the range of latency differences at a particular count difference is $\sim 30 \mathrm{~ms}$.) The leech behaviorally discriminated changes in latency difference with a threshold (75\% correct) detectable change in latency difference of $30 \mathrm{~ms}$ (Fig. 4D). At a latency difference change of just $6 \mathrm{~ms}$, the leech behaviorally discriminated the spike trains at 
$60 \%$ correct ( \pm 0.02 SEM $)$, which was significantly above chance ( $p=0.003$; onesided $t$ test; $n=11)$. From the encoding experiments, a latency difference change of $30 \mathrm{~ms}$ corresponds to a touch location change of $\sim 25^{\circ}$ (Fig. $2 E$ ).

Varying both count and latency difference We next changed both latency difference and count difference in the same preparation while monitoring the behavioral response. We used two latency differences, each paired with two count differences (Fig. 5A). In one set of experiments, we delivered variations in count difference and latency difference that simulated a change in touch location of $20^{\circ}$, which is about twice the behavioral threshold (Fig. $2 H)$. The results of one such experiment are shown in a scatter plot of the scores of the first three PCs of the behavioral response (Fig. 5B) [see Materials and Methods and Baca et al. (2005)]. This PC representation of the behavior preserves the metric structure of the original space of behavioral responses: two points that are far apart in this plot correspond to bend responses that are very dissimilar.

The results were qualitatively the same in all three experiments: the responses segregated into two clearly distinct clusters that corresponded to the two count differences, and within each of these clusters were two subclusters corresponding to the two latency differences applied. Quantitatively, the distance between the mean responses when the count difference was changed by three spikes was significantly greater than the distance between the mean responses when the latency difference was changed by $24 \mathrm{~ms}(p<0.001, t$ test; $n=6)$ (Fig. 5C). (We used distances between mean responses rather than percent correct because the discrimination of location was nearly perfect (100\% correct), so percent correct was not sensitive to differences in the response distributions.) We verified that this result holds for smaller variations in latency difference and count difference by changing the count difference by 1 spike and the latency difference by $10 \mathrm{~ms}$ (Fig. 5D) ( $p<0.05$, $t$ test; $n=11$ ).

Figure $5 E$ summarizes the results of the data from the decoding experiments. Changing the count difference (an integer) produces a relatively large discrete jump in the mean behavioral response (Fig. 5E, along axis $\mathrm{CD}$ ), whereas changing the latency difference (a continuous number) at a given count difference produces a small graded shift in the behavioral response (Fig. 5E, along axis LD). The direction of the shift in the behavioral response when the latency difference was changed was not correlated with the direction of the shift in the behavioral response when the count difference alone was changed $[p>0.05$, Rayleigh's test (Zar, 1999)] (Fig. 5E). This explains the unexpected result that the distance between the mean behavioral response when we changed both count difference and latency difference (stimuli represented by the points diagonally across from one another in Fig. 5A) did not significantly differ from the distance between the mean behavioral response when only count difference was changed (Fig. $5 C, D)(p>0.05 ; t$ test).

Count difference and latency difference have very different sensitivities in their ability to encode and decode (Fig. 5F). For
A2
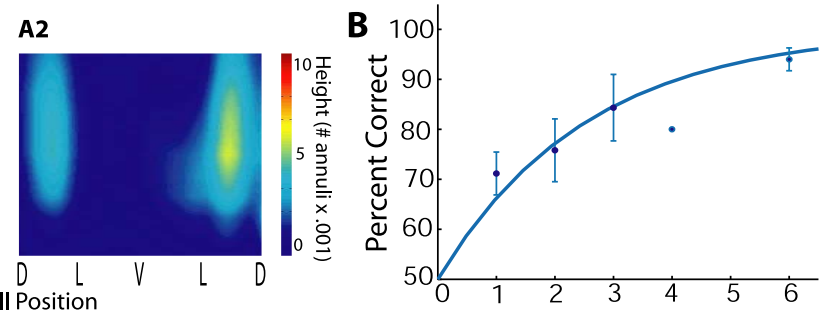

Count Difference Change (spikes)

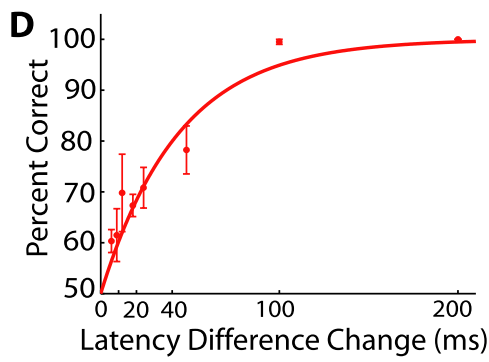

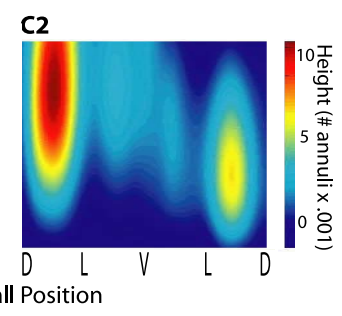

Latency Difference Change (ms)

Figure 4. Results of the decoding experiments. $\boldsymbol{A}$, Behavioral responses when the count difference was changed by four spikes liffere (ifference, $+12 \mathrm{~ms}$ ). In both $\mathbf{C}$ and $\mathbf{C}$, both $\mathbf{P}$ cells fired five spikes with the same instantaneous firing rates. $\boldsymbol{D}$, Percent correct

encoding, latency difference reaches threshold (i.e., 75\% correct) at a touch location separation of $4^{\circ}$, whereas count differences require a separation of $13^{\circ}$. However, for decoding, count difference reaches threshold at 1.8 spikes (which corresponds to a touch location difference of $14^{\circ}$ ), whereas latency difference reaches threshold at $30 \mathrm{~ms}$ (corresponding to a touch location separation of $25^{\circ}$ ). In other words, count difference is encoded coarsely and decoded precisely, whereas latency difference is encoded precisely and decoded coarsely.

\section{Discussion}

\section{Encoding versus decoding precision}

Whereas latency is an extremely precise encoder of touch location, and spike count is relatively imprecise, the converse was observed in the decoding experiments (Fig. $5 F$ ). There are at least two possible explanations of this negative correlation between encoding and decoding precision. First, it could be that response latency is not only used by the local bend network, but is a temporal code used by other leech behaviors that change on time scales smaller than the local bend response. Second, these differences in the sensitivity of encoding and decoding might arise because of preexisting constraints in the leech CNS. For example, because the transduction mechanism for spike count generation seems to be quite noisy, the decoding mechanisms for count differences would need to be very precise for count differences to be useful for directing local bending behavior. Conversely, if the leech motor neurons have extremely variable tonic firing rates that make them insensitive to input timing (Zoccolan et al., 2002), the encoding mechanism would need to be quite reliable to affect behavior.

Despite the fact that the decoding mechanisms are more sensitive to changes in count difference than latency difference, this does not imply that latency is unimportant in the control of the local bend response. Generally, both variables influence behavior 


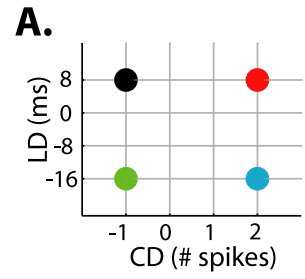

B.
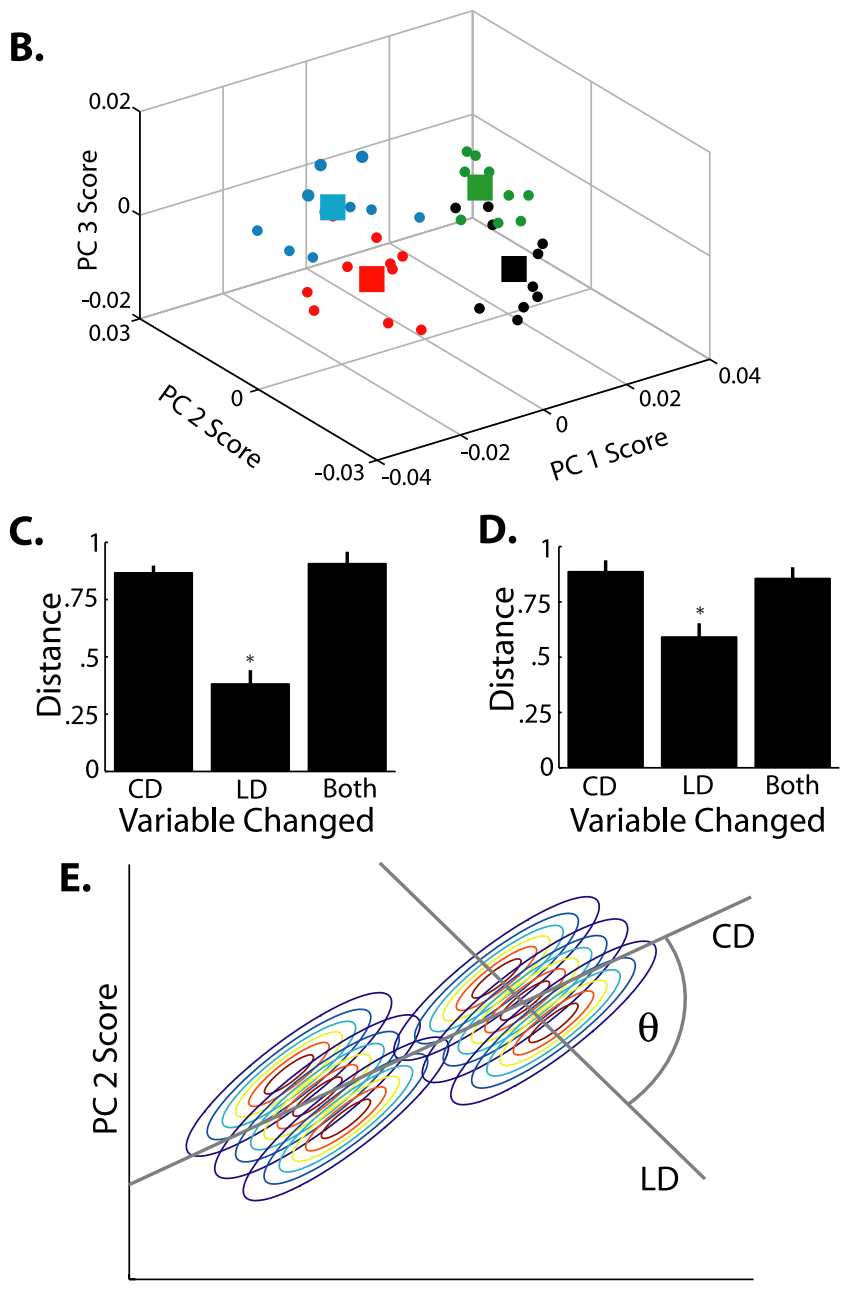

PC 1 Score

\section{F.}

\begin{tabular}{|l|c|c|}
\cline { 2 - 3 } \multicolumn{1}{c|}{} & Latency & Count \\
\hline \hline Encoding & $4^{\circ}$ & $13^{\circ}$ \\
\hline \hline Decoding & $25^{\circ}$ & $14^{\circ}$ \\
\hline
\end{tabular}

Figure 5. Results of decoding experiments in which we varied both latency difference and count difference. $A$, Illustration of the four classes of spike trains elicited in the P cells in one set of experiments. $C D$, Count difference; $L D$, latency difference. The first and second rows represent trials in which the latency difference was $+8 \mathrm{~ms}$ and $-16 \mathrm{~ms}$, respectively. The first and second columns represent trials in which the count difference was -1 spikes and +2 spikes, respectively. Hence, we generated count difference changes of three spikes (between columns), latency difference changes of $24 \mathrm{~ms}$ (between rows), as well as both changes (diagonals). $\boldsymbol{B}$, Results of one experiment in which we delivered the four stimuli represented in $\boldsymbol{A}$. The axes are $\mathrm{PC}$ scores and each point represents the bend response on a single trial. We use the same color code as in $\boldsymbol{A}$. The mean response to each stimulus is indicated by a large filled square. $\boldsymbol{C}$, Bar graph of the distance between mean behavioral responses when the different neuronal variables were changed (mean \pm SEM). Each distance within an experiment was normalized to the maximum distance between means for that experiment. " $C D$ " is the case in which count difference was changed by three spikes, " $L D$ " is the case in which latency difference was changed by $24 \mathrm{~ms}$, and "Both" indicates the case in which both variables changed. The asterisk indicates that the distance between the mean behavioral response to the latency difference change was significantly less than the distance when either count difference alone, or both within their range of values seen in vivo. For instance, in the encoding experiments (Fig. 2), we observed that the count difference was quite variable, so that the touch location could change by as much as $30^{\circ}$ without an accompanying change in the count difference. The latency difference in this case would be sufficient to cause the leech to respond differently to the two stimuli. Indeed, we found that latency difference changes as small as $6 \mathrm{~ms}$ (which corresponds to a touch-location difference of $\sim 5^{\circ}$ ) were behaviorally discriminated above chance.

\section{Latency: a universal location code?}

Attempts to explain touch location discrimination have typically relied on count-based sensory codes (Sur et al., 1980; Wheat et al., 1995) (but see Jones, 1956; Johansson and Birznieks, 2004). For instance, locations on the body where touch location discrimination is most acute are represented in the primary somatosensory cortex with more neurons that have smaller count-based receptive fields (Sur et al., 1980; Wheat et al., 1995). Whereas countbased codes probably partly explain mammalian touch localization, this increased cortical representation could also provide more neurons for a higher-fidelity temporal code. Recent converging lines of evidence recommend this hypothesis. First, in most sensory systems, the time of the first spike after a stimulus (i.e., the response latency) is often particularly informative about stimulus value (Hartline, 1938; Gawne et al., 1996; Eggermont, 1998; Raiguel et al., 1999; Reich et al., 2001; Oram et al., 2002). Most relevantly, stimulus location is encoded by the relative response latency in populations of cortical neurons in the primary somatosensory cortex of anesthetized rats (Petersen et al., 2002; Foffani et al., 2004), and in humans, response latency in individual peripheral afferents encodes touch location (Johansson and Birznieks, 2004). It would be quite surprising if the relative latencies in populations of such afferents did not encode touch location; the experiment has yet to be done. In view of such data, the impressive capacity relative latency has for encoding stimulus parameters [long recognized in theoretical work (Thorpe, 1990)], and the results presented here, we expect that relative latency is a primitive feature of somatosensory location encoding that is preserved across multiple phyla.

\section{Future directions and open questions}

What is the cause of the observed latency-difference code in leech mechanoreceptors? Two possibilities suggest themselves. (1) As the stimulus moves further from the receptive field center of a $\mathrm{P}$ cell (Fig. $1 B$ ), the threshold stimulation required for spike initiation increases, so the same stimulus will tend to evoke spikes

$\leftarrow$

variables, were changed ( $p<0.001, t$ test; $n=6)$. D, Same as in $\boldsymbol{C}$, except the count difference change was 1 spike and the latency difference change was $10 \mathrm{~ms}(p<0.05, t$ test; $n=11) . \boldsymbol{E}$, Summary of how the behavioral response (in PC-score space) is affected by changes in count difference and latency difference. The distribution of responses at a fixed count and latency difference are represented by contour plots of Gaussian distributions (the Gaussian was arbitrarily chosen for illustrative purposes). Within the set of responses at a particular count difference, there is a small graded change in the mean response as latency difference is changed (along axis $\mathrm{LD}$ ). When the count difference is changed, there is a marked jump in the mean response (along axis $(D)$. $\theta$ represents the angle between the $C D$ and $L D$ axes (for discussion, see Results). $\boldsymbol{F}$, Table comparing the performance of latency difference and count difference (columns) in the encoding and decoding experiments (rows). For the encoding row, the threshold touch location change required to achieve threshold discrimination ( $75 \%$ correct) is shown. The decoding row shows the change in the neuronal variable required to achieve threshold behavioral discrimination of that variable in the decoding experiments (after conversion to the distance between stimuli that would evoke such changes in the neuronal variable; see Results). 
with greater latency. (2) The latency could be a function of the cable length between transducers in the skin and the somata of the $\mathrm{P}$ cells, so the latencies observed are explained by conduction time from transducer to soma. We favor the second, conductiontime difference hypothesis because preliminary data have shown that stimulating the same patch of skin at different intensities does not significantly change the latency of the P-cell response (S. Baca, personal communication).

One limitation of the present study is that, for technical reasons, we could not record from $\mathrm{P}$ cells, mechanically stimulate the body wall, and acquire images of the body wall at the same time. Specifically, we could not use the force generator and electrophysiological setup simultaneously without occluding the leech body wall from our camera. It would be extremely useful if future researchers could overcome these technical limitations to discern, on a trial-by-trial basis, correlations among stimulus, P-cell activity, and behavior.

There were two important differences between the behavioral experiments that showed that the leech can discriminate touch location changes of $9^{\circ}$ (see Materials and Methods) (Baca et al., 2005), and the decoding experiments in the present paper. First, in the decoding experiments the body wall was cut and pinned slightly differently than in the behavioral experiments (see Materials and Methods). Although the bend response was qualitatively the same in the two body-wall preparations, these subtle differences in the dissection could introduce quantitative differences between the responses in the two cases. Second, in addition to the $\mathrm{P}$ cells, there is a class of mechanoreceptors known as "T cells" ( $\mathrm{T}$ stands for touch) (Kristan, 1982; Lewis and Kristan, 1998c) that would have been activated in the behavioral experiments, and we did not stimulate them in the decoding experiments. Although previous studies indicate that $\mathrm{T}$ cells have only a small modulatory effect on the local bend response (Kristan, 1982; Lewis and Kristan, 1998c; Zoccolan and Torre, 2002), this issue could be profitably re-examined using the techniques of the present paper.

Because of the simplicity of the leech CNS and our ability to generate arbitrary spike trains in the $\mathrm{P}$ cells while observing local bending, we have been able to test whether two of the variables that encode touch location are actually decoded in an intact circuit that includes all elements from the stimulus to the behavioral response. We have explored only a small region of the enormous parameter space that the technique has opened for exploration. We focused on spike counts and latencies because the coding data showed that both were strongly correlated with touch location, but there are many other possible parameters (such as the instantaneous firing rate, correlations between spike trains from two $\mathrm{P}$ cells (Perkel and Bullock, 1968; König and Engel, 1995), and the duration of stimulation) that can and should be examined in future studies. Furthermore, the same questions about encoding and decoding that we have experimentally addressed in the sensory neurons should be attacked at the level of interneurons and motor neurons.

\section{References}

Amassian VE (1953) Evoked single cortical unit activity in the somatic sensory areas. Electroencephalogr Clin Neurophysiol Suppl 5:415-438.

Baca SM, Thomson EE, Kristan WB (2005) Location and intensity discrimination in the leech local bend response quantified using optic flow and principal components analysis. J Neurophysiol 93:3560-3572.

Britten K, Shadlen MN, Newsome WT, Movshon JA (1992) The analysis of visual motion: a comparison of neuronal and psychophysical performance. J Neurosci 12:4745-4767.

Cholewiak RW (1999) The perception of tactile distance: influences of body site, space, and time. Perception 28:851-875.

Duda RO, Hart PE, Stork DG (2000) Pattern classification. New York: Wiley-Interscience.
Efron B, Tibshirani RJ (1994) An introduction to the bootstrap. Boca Raton, FL: Chapman and Hall/CRC.

Eggermont JJ (1998) Azimuth coding in primary auditory cortex of the cat. II. Relative latency and interspike interval representation. J Neurophysiol 80:2151-2161.

Foffani G, Tutunculer B, Moxon KA (2004) Role of spike timing in the forelimb somatosensory cortex of the rat. J Neurosci 24:7266-7271.

Gawne TJ, Kjaer TW, Richmond BJ (1996) Latency: another potential code for feature binding in striate cortex. J Neurophysiol 76:1356-1360.

Hartline HK (1938) The response of single optic nerve fibers of the vertebrate eye to illumination of the retina. Am J Physiol 121:400-415.

Johansson RS, Birznieks I (2004) First spikes in ensembles of human tactile afferents code complex spatial fingertip events. Nat Neurosci 7:170-177.

Johnson KO, Phillips JR (1981) Tactile spatial resolution. I. Two-point discrimination, gap detection, grating resolution, and letter recognition. J Neurophysiol 46:1177-1191.

Jones FN (1956) Space-time relationships in somesthetic localization. Science $124: 484$.

Kohonen T (1995) Self-organizing maps. Berlin: Springer.

König P, Engel AK (1995) Correlated firing in sensory-motor systems. Curr Opin Neurobiol 5:511-519.

Kristan WB (1982) Sensory and motor neurones responsible for the local bending response in leeches. J Exp Biol 96:161-180.

Kristan WB, McGirr SJ, Simpson GV (1982) Behavioural and mechanosensory neurone responses to skin stimulation in leeches. J Exp Biol 96:143-160.

Lewis JE, Kristan WB (1998a) A neuronal network for computing population vectors in the leech. Nature 391:76-79.

Lewis JE, Kristan WB (1998b) Quantitative analysis of a directed behavior in the medicinal leech: implications for organizing motor output. J Neurosci 18:1571-1582.

Lewis JE, Kristan WB (1998c) Representation of touch location by a population of leech sensory neurons. J Neurophysiol 80:2584-2592.

Lockery SR, Kristan WB (1990) Distributed processing of sensory information in the leech. II. Identification of interneurons contributing to the local bending reflex. J Neurosci 10:1816-1819.

Oram MW, Xiao DK, Dritschel B, Payne KR (2002) The temporal precision of neural signals: a unique role for response latency? Philos Trans R Soc Lond B Biol Sci 357:987-1001.

Parker AJ, Newsome WT (1998) Sense and the single neuron: probing the physiology of perception. Annu Rev Neurosci 21:227-277.

Perkel DH, Bullock TH (1968) Neural coding. Neurosci Res Program Bull 6:221-348

Petersen RS, Panzeri S, Diamond ME (2001) Population coding of stimulus location in rat somatosensory cortex. Neuron 32:503-514.

Petersen RS, Panzeri S, Diamond ME (2002) Population coding in somatosensory cortex. Curr Opin Neurobiol 12:441-447.

Raiguel SE, Xiao D, Marcar VL, Orban GA (1999) Response latency of macaque area MT/V5 neurons and its relationship to stimulus parameters. J Neurophysiol 82:1944-1956.

Reich DS, Mechler F, Victor JD (2001) Temporal coding of contrast in primary visual cortex: when, what, and why? J Neurophysiol 85:1039-1050.

Sur M, Merzenich MM, Kaas JH (1980) Magnification, receptive-field area, and "Hypercolumn" size in Areas $3 \mathrm{~b}$ and 1 of somatosensory cortex in owl monkeys. J Neurophysiol 44:295-311.

Thomson EE, Kristan WB (2005) Quantifying stimulus discriminability: a comparison of information theory and ideal observer analysis. Neural Computation 17:741-778.

Thorpe SJ (1990) Spike arrival times: a highly efficient coding scheme for neural networks. In: Parallel processing in neural systems and computers, (Eckmiller R, ed), pp 91-94. Dusseldorf, Germany: Elsevier.

Wheat HE, Goodwin AW, Browning AS (1995) Tactile resolution: peripheral neural mechanisms underlying the human capacity to determine positions of objects contacting the fingerpad. J Neurosci 15:5582-5595.

Zar JH (1999) Biostatistical analysis, Ed 4. Upper Saddle River, NJ: Prentice Hall.

Zoccolan D, Torre V (2002) Using optical flow to characterize sensorymotor interactions in a segment of the medicinal leech. J Neurosci 22:2283-2298.

Zoccolan D, Pinato G, Torre V (2002) Highly variable spike trains underlie reproducible sensorimotor responses in the medicinal leech. J Neurosci 22:10790-10800. 